\title{
Promoting predictive, preventive and personalized medicine in treatment of cardiovascular diseases
}

\author{
Hiroyasu Iso
}

Published online: 7 April 2011

(C) European Association for Predictive, Preventive and Personalised Medicine 2011

The field of cardiovascular diseases is one of the major targets for predictive, preventive and personalized medicine because there is a large body of evidence concerning cardiovascular risk factors and preventive strategies at both population and individual levels. The present issue of The EPMA Journal focuses on predictive, preventive and personalized medicine for cardiovascular diseases and reviews the broad aspects of basic, clinical and epidemiological researches. There are thirteen articles, seven from European countries (Germany, UK, Italy, Denmark and Czech Republic), one from the United States and five from Japan. The contributing countries are shown in Fig. 1.

With regard to predictive medicine, Professor Hana Rosolova and Dr. Barbora Nussbaumerova (Czech Republic) presents a comprehensive review of risk factors for cardiovascular diseases and prevention strategies; the data were derived primarily from epidemiological studies in the Europe and the United States. Professor Rosolova stresses the importance of cardio-metabolic risk factor assessment for the primary prevention of cardiovascular diseases [1]. Dr. Kenneth J. Colley et al. (USA) points out that lipoprotein-associated phospholipase $A_{2}$, a vascular-specific inflammation marker, is useful for refining cardiovascular risk assessment, especially for individuals at intermediate cardiovascular risk [2].

A series of articles by Japanese scientists reviews risk factors for cardiovascular diseases from an Asian perspective and shows similarities and differences in risk factors

H. Iso is a National Representative of EPMA in Japan.

H. Iso $(\bowtie)$

Professor of Public Health,

Department of Social and Environmental Medicine,

Osaka University Graduate School of Medicine,

2-2 Yamadagaoka, Suita-shi,

Osaka 565-0871, Japan

e-mail: iso@pbhel.med.osaka-u.ac.jp compared with those reported for Europe and the United States [3-6]. Professor Katsuyuki Miura (Japan) stresses that high blood pressure is the strongest risk factor for stroke, and that a major decline in blood pressure levels has been contributing to a drastic reduction in stroke mortality since the $1960 \mathrm{~s}$ to make Japan the country with the longest longevity [3]. However, other health problems have been emerging, such as overweight, impaired glucose metabolism and dyslipidemia.

Dr. Hiroshi Yatsuya et al. (Japan) address the increasing prevalence of overweight among middle-aged men but not women, which may explain in part why the incidence of ischemic stroke and ischemic heart disease has stopped declining or even shown a tendency to increase [4]. Overweight was found to be associated with risk of ischemic stroke for both men and women, and risk of ischemic heart disease for men only. They also addresses two important continuing health issues for Japanese, namely, being at high cardiovascular risk (e.g. due to hypertension) without being overweight or being obese which should be compensated for by anti-overweight strategies.

Dr. Yoshihiro Kokubo (Japan) reviews the involvement of glucose abnormality and hyperlipidemia in risk of cardiovascular diseases among Japanese, and presents similarities and differences in the contributions of these two risk factors [5]. The similarities are high levels of total and LDL-cholesterol or of triglycerides as a risk factor for ischemic stroke (atherothrombotic infarction) and ischemic heart disease, and low levels of HDL-cholesterol as a risk factor for the same outcomes, while the difference consists of low levels of total or LDL-cholesterol as a potential risk factor for intracerebral (intraparenchymal) hemorrhage. Dr. Kokubo's group's study shows that impaired glucose metabolism could be an emerging risk factor for ischemic heart disease in women. 


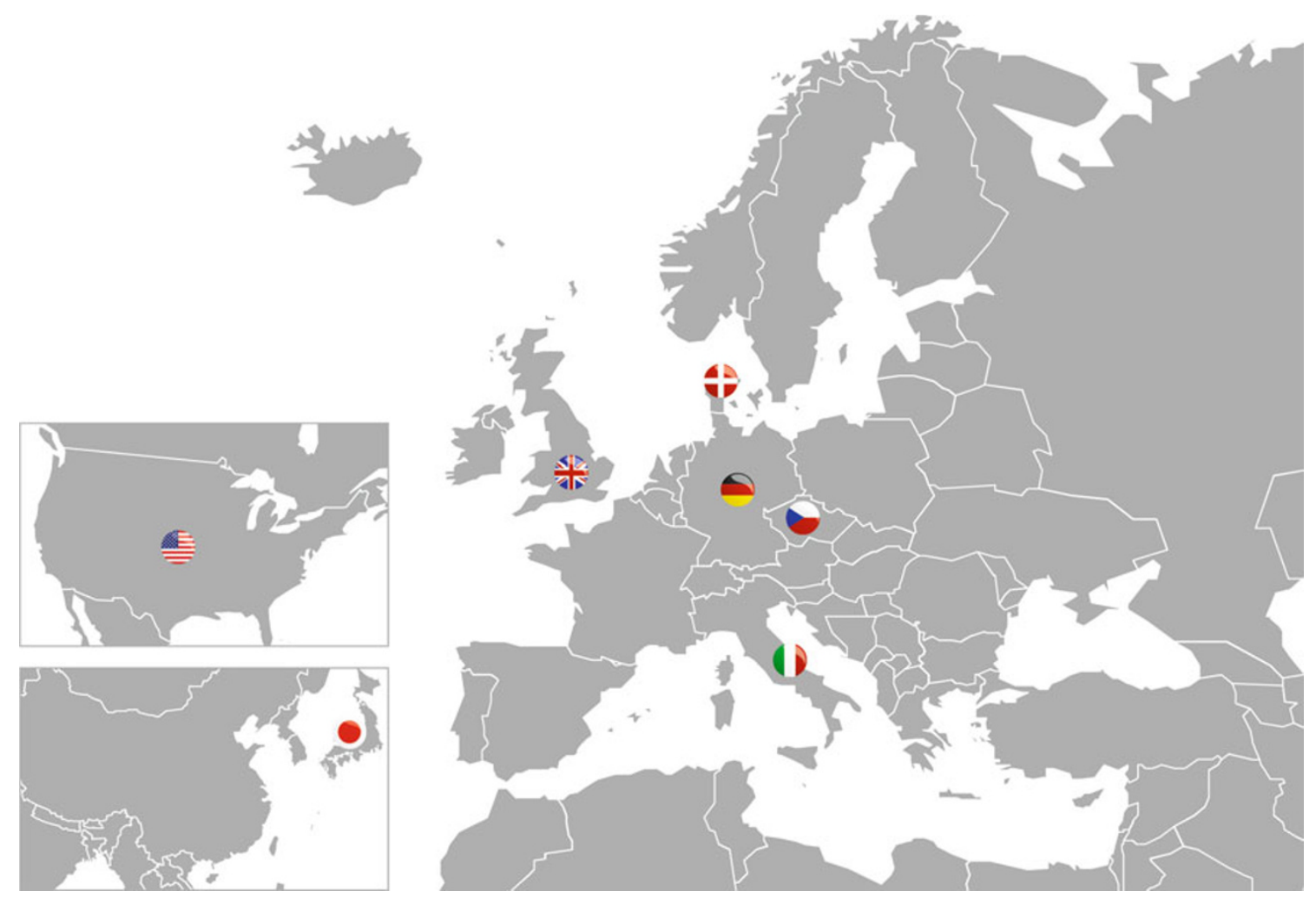

Fig. 1 Map showing the countries of the contributors to the editorial and review articles in the current journal issue

The article by Hiroyasu Iso overviews long-term trends in cardiovascular diseases and their risk factors in Japan from the beginning of the $1960 \mathrm{~s}$ until the $2000 \mathrm{~s}$ [6]. The net effects of declining blood pressure and increasing dyslipidemia in both men and women, and of reduced smoking and increases in overweight and glucose abnormality in men were major declines in stroke and ischemic heart disease for both men and women, although ischemic heart disease in urban men showed a tendency to increase. These dynamic changes in cardiovascular risk factors may have caused changes in the relative importance of underlying two vascular pathologies, i.e. small vessel pathology (arteriosclerosis, causing primarily cerebral hemorrhage and lacunar infarction) and large vessel pathology (atherosclerosis, causing primarily ischemic heart disease), from dominance of small vessel pathology to a balance of small and large vessel pathologies.

As for preventive medicine, the Japanese experience is described as a health success story [6]. The combination of improvements in public health and personalized treatment activities has contributed to reductions in sodium intake, more balanced diets, reduction or cessation of smoking as well as enhanced hypertension detection and treatment. Evidence is presented that our community-based stroke prevention program resulted in a reduction in the incidence and prevalence of stroke. However, potential, future health threats remain, that is, plateauing of mortality decline and an increasing incidence of ischemic heart disease among urban middle-aged men, probably due to an increase in dyslipidemia and diabetes mellitus.

Dr. Paul C. Evans (UK) discusses the potential benefit of sulforaphane, an isothiocyanate derived from green vegetables, for the attenuation of atherosclerosis development thorough anti-inflammation and anti-oxidant activities [7]. Investigation of the mechanisms underlying this potential benefit is expected to inform new nutritional approaches to the prevention of vascular inflammation and atherosclerosis.

Obstructive sleep apnea, an emerging risk factor for atherosclerosis and cardiovascular diseases, is discussed by Dr. Izabela Tuleta et al. (Germany), and Professor Takeshi Tanigawa (Japan) $[8,9]$. Dr. Tuleta et al. address mechanisms linking obstructive sleep apnea with atherosclerosis, and extensively review epidemiological and clinical studies on obstructive sleep apnea (or CPAP therapy) and atherosclerosis/cardiovascular disease [8]. Professor Tanigawa reports on his invention of the single-channel airflow monitor, a new device for the measurement of nocturnal intermittent hypoxia [9]. He also expands on the epidemiological evidence for the relationship between obstructive sleep apnea and cardiovascular risk factors such as hypertension, atrial fibrillation, C-reactive protein, metabolic syndrome and Type 2 diabetes among Japanese. He further states that screening for obstructive sleep apnea with his new device or by 
pulse oximetry seems promising for the detection and control of this disorder.

As for personalized medicine, Professor Jens Kastrup (Denmark) and Dr. Ulrich M. Becher et al. (Germany) provide an overview of the present situation concerning innovative stem-cell treatment for cardiovascular diseases and its perspectives $[10,11]$. Professor Kastrup comprehensively reviews the outcomes of clinical stem-cell treatments for ischemic heart disease and heart failure [10]. The results are inconsistent, however, and little information is available on the efficacy of various cell types. Ongoing or planned larger randomized clinical trials are expected to provide answers for some of the questions regarding optimal cell types, numbers, cell sources and delivery methods. Dr. Becher focuses more on the mechanisms of action by stem cells and addresses intra-individual variations in the outcomes of stem cell treatments [11]. Stem cell treatment is one of the most promising approaches for the treatment of patients with ischemic heart disease and heart failure. Further extensive research is needed, however, to optimize the treatment effects, which depend on individual patient's factors, in order to aid in the establishment of personalized regenerative medicine.

Dr. Kristina Yeghiazaryan (Germany) et al. reviews clinical and molecular aspects of degenerative processes in aortic valves, which lead to aortic stenosis, the most common valvular disease. Exploration of molecular markers and clinical risk factors for this degenerative process may enhance the performance of predictive and preventive measures [12].

Drs. Paolo Roberti di Sarsina and Haria Iseppato (Italy) stress the importance of and need for integrative medicine, i.e., the integration of modern western medicine and other cheaper, more holistic, more person-centered medicine [13].

Finally, the promotion of predictive, preventive and personalized medicine in the treatment of cardiovascular diseases is a global health issue because the health burden from cardiovascular diseases is currently the most severe in most developed countries and is rapidly increasing in most developing countries [14]. It is therefore of the utmost importance to exchange on global-level scientific insights, knowledge and skills for risk prediction of cardiovascular diseases, and to share and adopt various experiences for its prevention and the development of personalized medicine. The current issue of The EPMA Journal provides an excellent platform for this challenge.

\section{References}

1. Rosolova H, Nussbaumerova B. Cardio-metabolic risk prediction should be superior to cardiovascular risk assessment in primary prevention of cardiovascular diseases. EPMA J. 2011. doi:10.1007/s13167-011-0066-1.
2. Colley KJ, Wolfert RL, Cobble ME. Lipoprotein associated phospholipase A2: role in atherosclerosis and utility as a biomarker for cardiovascular risk. EPMA J. 2011. doi:10.1007/ s13167-011-0063-4.

3. Miura K. Epidemiology and prevention of hypertension in Japanese: how could Japan get longevity? EPMA J. 2011. doi:10.1007/s13167-011-0069-y.

4. Yatsuya H, Yamagishi Y, Iso H. Adiposity and risk of cardiovascular diseases in Japan: secular trend, individual level associations and causal pathway - implications for the prevention of cardiovascular diseases in societies with rapid economic development. EPMA J. 2011. doi:10.1007/s13167-011-0071-4.

5. Kokubo Y. Associations of impaired glucose metabolism and dyslipidemia with cardiovascular diseases: what have we learned from Japanese cohort studies for individualized prevention and treatment? EPMA J. 2011. doi:10.1007/s13167-011-0074-1.

6. Iso H. A Japanese health success story: trends in cardiovascular diseases, their risk factors, and the contribution of public health and personalized approaches. EPMA J. 2011. doi:10.1007/ s13167-011-0067-0.

7. Evans PC. The influence of sulforaphane on vascular health and its relevance to nutritional approaches to prevent cardiovascular disease. EPMA J. 2011. doi:10.1007/s13167-011-0064-3.

8. Tuleta I, Pabst S, Juergens UR, Nickenig G, Skowasch D. Obstructive sleep apnoea as a risk factor for atherosclerosis implication for preventive and personalised treatment. EPMA J. 2011. doi:10.1007/s13167-011-0070-5.

9. Tanigawa T. Obstructive sleep apnea: its prevention and screening may contribute to the prevention of hypertension, diabetes and cardiovascular diseases. EPMA J. 2011. doi:10.1007/s13167-0110073-2.

10. Kastrup J. Stem cells therapy for cardiovascular repair in ischemic heart disease: How to predict and secure optimal outcome? EPMA J. 2011. doi:10.1007/s13167-011-0062-5.

11. Becher UM, Tiyerilli V, Skowasch D, Nickenig G, Werner N. Personalized cardiac regeneration by stem cells - Hype or hope? EPMA J. 2011. doi:10.1007/s13167-011-0068-z.

12. Yeghiazaryan K, Skowash D, Bauriedel G, Schild HH, Golubnitschaja O. Degenerative valve disease and bioprostheses: risk assessment, predictive diagnosis, personalised treatments. EPMA J. 2011. doi:10.1007/s13167-011-0072-3.

13. Roberti di Sarsina P, Iseppato I. Why we need integrative medicine. EPMA J. 2011. doi:10.1007/s13167-011-0065-2.

14. Reddy KS, Yusuf S. Emerging epidemic of cardiovascular disease in developing countries. Circulation. 1998;97:596-601.

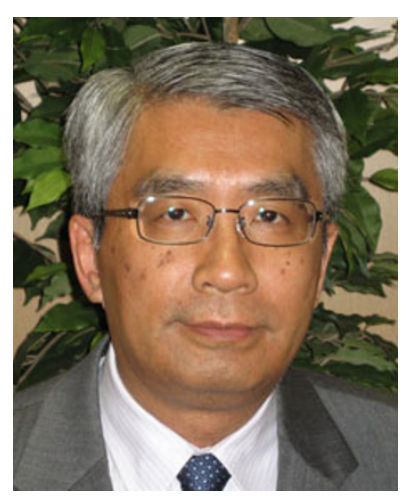

Hiroyasu Iso is Professor of Public Health, Department of Social and Environmental Health, Osaka University Graduate School of Medicine. He graduated from medical school in 1982 and received his DMS (Doctor of Medical Science) in 1986 from the University of Tsukuba, and his MPH in 1988 from the University of Minnesota under the Fulbright Scholarship program. He worked as visiting Associate Professor of Medicine at Harvard University in 1996-1997, and assumed his present position as Professor of Public Health in 2005. His major interests are epidemiology and prevention of lifestyle related diseases and he has published over 350 original articles in these areas. He has directed public health courses in the 
master's program of Medical Science at Osaka University since 2007. $\mathrm{He}$ received a grant from the Japan Science and Technology Agency for sending 27 medical undergraduate, graduate and postgraduate students overseas to study social epidemiology at topranking universities (Harvard University, University College of London and the Karolinska Institute) between 2010 and 2012. This project has shown great potential to make a major contribution to the development of social epidemiology in Asia. Professor Iso has also directed a large national birth cohort study conducted in Japan by a research center at Osaka University. This cohort is composed of 15 nationwide unit centers covering 100,000 births, which were followed up until the age of 13 for a comprehensive investigation of the influence of genetic, environmental and psychosocial factors on children's development and health. He serves as a trustee for the Japan Public Health Association, the Japan Epidemiological Association, and the Japan Society of Preventive Cardiology, and is the editor-in-chief of the Journal of Epidemiology and an editor of The EPMA Journal. 